\title{
Costs of medication in older patients: before and after comprehensive geriatric assessment
}

This article was published in the following Dove Press journal:

Clinical Interventions in Aging

\author{
Gulcin Done Unutmaz' \\ Pinar Soysal ${ }^{2}$ \\ Busra Tuven' \\ Ahmet Turan Isik ${ }^{3}$ \\ 'Department of Internal Medicine, \\ Faculty of Medicine, Dokuz Eylul \\ University, Izmir, Turkey; ${ }^{2}$ Geriatric \\ Center Kayseri Education and \\ Research Hospital, Kayseri, Turkey; \\ ${ }^{3}$ Department of Geriatric Medicine, \\ Faculty of Medicine, Dokuz Eylul \\ University, Izmir, Turkey
}

Background: Polypharmacy and inappropriate drug use cause numerous complications, such as cognitive impairment, frailty, falls, and functional dependence. The present study aimed to determine the effect of the comprehensive geriatric assessment (CGA) on polypharmacy, potentially inappropriate medications (PIMs) and potential prescribing omissions (PPOs), and to evaluate the economic reflections of medication changes.

Methods: One thousand five hundred and seventy-nine older patients, who had undergone CGA, were retrospectively evaluated. The drugs, drug groups, and number of drugs that the patients used were recorded. Appropriate drug therapy was identified by both CGA and STOPP/ START criteria. Based on these criteria, PIMs were discontinued and PPOs were started. The monthly cost of these drugs was calculated separately for PIMs and PPOs by using the drugstore records.

Results: After CGA, while the prevalence of non-polypharmacy was increased from $43.3 \%$ to $65.6 \%$, the prevalence of polypharmacy and hyperpolypharmacy was decreased from $56.7 \%$ to $34.4 \%$ and $12.0 \%$ to $3.6 \%$, respectively. The three most common PIMs discontinued were proton pump inhibitors, anti-dementia drugs, and antipsychotics, respectively. However, the most common PPOs started were vitamin D and B12 supplements, and anti-depressants. After CGA, monthly saved total per capita cost of PIMs was US\$12.8 and monthly increased total per capita cost of PPOs was $\$ 5.6$.

Conclusion: It was demonstrated that prevalence of polypharmacy, PIM, and PPO could be decreased by CGA including START/STOPP criteria in older adults. Furthermore, this will have beneficial effects on economical parameters due to decreasing drug-related health care costs.

Keywords: cost, comprehensive geriatric assessment, polypharmacy, elderly

\section{Introduction}

Comorbidities and use of multiple medications increase with advancing age; this may cause significant drug-related complications such as polypharmacy and increased health care costs. ${ }^{1}$ Guidelines on treatment of chronic diseases are inadequate in older patients with multiple comorbidities. ${ }^{2}$ In addition, alterations in drug pharmacokinetics and pharmacodynamics in patients with advancing age make effective and safe pharmacotherapy difficult in these patients. ${ }^{3}$ As shown by the World Health Organization, polypharmacy has become a critical public health problem due to the growth of the aging population. ${ }^{1-3}$ It is known that polypharmacy and inappropriate drug use cause numerous complications, such as cognitive deficiency, frailty, urinary incontinence, depression, malnutrition, balance and gait disorders, falls, and reduced functional capacity, and increase the frequency of living in nursing homes, hospital admissions, and hospital stay. ${ }^{1-3}$ Therefore, combating polypharmacy in geriatric practice is crucial to reduce health care costs.
Correspondence: Ahmet Turan Isik Dokuz Eylul University, School of Medicine, 35340 Balcova, Izmir, Turkey Email atisik@yahoo.com

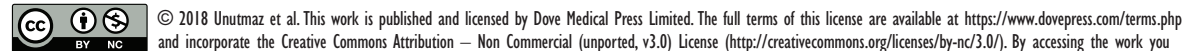
BY NC and incorporate the Creative Commons Attribution - Non Commercial (unported, v3.0) License (http:///creativecommons.org/licenses/by-nc/3.0/). By accessing the work you
hereby accept the Terms. Non-commercial uses of the work are permitted without any further permission from Dove Medical Press Limited, provided the work is properly attributed. For permission for commercial use of this work, please see paragraphs 4.2 and 5 of our Terms (https://www.dovepress.com/terms.php). 
In the early 1990s, Johnson and Bootman demonstrated that an average of US\$76 billion was spent annually in the USA to solve drug-related problems and that drug-related hospitalization accounted for the majority of this amount. This cost was updated to US\$177.4 billion. ${ }^{4,5}$ In another study, the total cost of morbidity and mortality due to inappropriate drug use was reported to be higher than the cost of drugs. ${ }^{6,7}$ Considering that these studies comprised patients of all age groups, it is probable that costs will be much higher when only older patients are evaluated. On account of all the aforementioned reasons, polypharmacy is a serious public health problem and its prevention should be approached as a health policy.

The comprehensive geriatric assessment (CGA) has an important role in assessing polypharmacy in geriatric patients. ${ }^{8}$ It is possible to determine and optimize the potentially inappropriate medications (PIMs) and potential prescribing omissions (PPOs) by the inclusion of the screening tool of older people's prescriptions (STOPP) and the screening tool to alert to right treatment (START) criteria in this assessment. ${ }^{9}$

The present study aimed to identify comorbidities of patients who presented to the geriatrics outpatient clinic of a tertiary hospital and drugs they used; to determine the effect of the CGA on polypharmacy, PIM and PPO; to detect the drug groups, included in and excluded from the treatment, by the CGA; to identify inappropriate drug use in clinical practice; and to evaluate the economic reflections of changes to be performed in the number of drugs used in treatment.

\section{Methods}

\section{Study design and participants}

Hospital files of 1,942 patients, who attended to the geriatrics outpatient clinic between January 2014 and February 2016 for any reason and underwent the CGA, were retrospectively reviewed. Only 1,579 patients without missing information in their hospital files were included. The study was approved by Dokuz Eylul University Local Ethics Committee. Since all data presented in this retrospective study were de-identified, the patient consent statement was not required. There were no exclusion criteria for the study.

\section{Data collection and CGA}

Sociodemographic data (age, gender, education status, and living environment) and history of chronic diseases (hypertension, diabetes mellitus, coronary artery disease, cerebrovascular disease, congestive heart failure, hyperlipidemia, peripheral vascular disease, depression, dementia, hypothyroidism, osteoporosis, COPD) were recorded. In addition, comorbidity status of the patients was evaluated using the Charlson Comorbidity Index (CCI). To evaluate cognitive functions, the Cognitive State Test, the Mini-Mental State Examination (MMSE), and the Montreal Cognitive Assessment (MoCA) scales were given to those who were illiterate older people, to those who had education under 11 years, and had education over 11 years, respectively. The CGA that the patients underwent included the Yesavage Geriatric Depression Scale (YGDS), the Lawton-Brody Instrumental Activities of Daily Living (IADL) scale, the Barthel Index (BADL); Up\&Go scale, Tinetti Performance Oriented Mobility Assessment (POMA) for balance (POMA-B) and gait (POMA-G), and the Mini-Nutritional Assessment (MNA). ${ }^{10,11}$

Laboratory tests performed to assess biochemical, metabolic, and nutritional status of the patients included measurements of complete blood count, kidney and liver functions, cholesterol levels, thyroid stimulating hormone, HbA1c, and levels of vitamin B12, folic acid, and vitamin D. All of these biochemical tests were performed using the Diagnostic Modular Systems auto analyzer (Roche E170 and P-800).

\section{Data on polypharmacy and drugs}

The drugs, drug groups, and number of drugs that the patients used were recorded. Using five or more drugs was considered polypharmacy and using ten or more drugs was considered hyperpolypharmacy. ${ }^{12}$ Appropriate drug use of the patients was identified by CGA complemented with STOPP/START criteria. ${ }^{9}$ Based on these criteria, PIMs were discontinued and PPOs were determined by a geriatrician; thus, treatments of the patients were rearranged. The drugs, drug groups, and number of drugs in the new treatments were also evaluated. Data on the drugs before and after applying CGA were compared.

\section{Cost analysis}

Drugs that were discontinued and included in the treatment after CGA were recorded. Using the drugstore records, monthly retail cost corresponding to the optimum dose of these drugs and the mean per capita cost to the government were quantified separately for PIMs and PPOs.

\section{Statistical analysis}

All statistical analyses were performed using the Statistical Package for the Social Sciences version 15.0 (SPSS Inc., Chicago, IL, USA). Parametric values were expressed as mean $\pm \mathrm{SD}$, data obtained from the scoring systems were expressed as median (interquartile range), and categorical 
values were expressed as percentage. Student's $t$-test was used for the comparison of normally distributed parametric data, whereas the Mann-Whitney $U$ test and the Wilcoxon test were used for the comparison of not-normally distributed data. Categorical data were analyzed by chi-square test. A $p$-value of $<0.05$ was considered significant.

\section{Results}

In the present study, 1,579 patients were included. Polypharmacy was identified in 895 (56.7\%) patients and nonpolypharmacy was identified in $684(43.3 \%)$ patients. Hyperpolypharmacy was found in 190 (12.0\%) patients. After CGA, non-polypharmacy, polypharmacy, and hyperpolypharmacy were found in $65.6 \%(1,036), 34.4 \%$ (543), and $3.6 \%(57)$, respectively. Mean number of drugs at first examination was $5.3 \pm 3.4$, and after CGA, mean number of drugs decreased to $4.6 \pm 2.5(p<0.05)$.

Polypharmacy and non-polypharmacy groups were compared in terms of sociodemographic characteristics, CGA parameters, and laboratory findings. In the polypharmacy group, the mean age, frequency of falls, education level, and body mass index were significantly lower than those in the non-polypharmacy group $(p<0.05)$. However, the CCI score and the presence of diabetes mellitus, hyperlipidemia, hypertension, cerebrovascular disease, depression, COPD, congestive heart failure, coronary artery disease, and dementia was significantly more prevalent in the polypharmacy group $(p<0.05)$. In addition, while the scores of the MMSE, MoCA, MNA, IADL, BADL, POMA-B, and POMA-G were significantly lower in the polypharmacy group, the YGDS and Up\&Go scores were significantly higher $(p<0.05)$. Compared with the non-polypharmacy group, in the polypharmacy group, the serum albumin level was significantly lower; however, serum vitamin B12 level was significantly higher $(p<0.05)$ (Table 1). When the age and education effect was eliminated, there were still significant differences between the groups in terms of diabetes mellitus, vitamin B12 level, and the scores of the IADL, BADL, POMA-B, POMA-G, and Up\&Go scale $(p<0.05)$. Among the patients, $8.3 \%$ had no comorbidity and the rates of patients with $1,2,3,4,5$, and $>5$ comorbidities were $14.3 \%, 23.7 \%, 22.8 \%, 14.8 \%$, $9.5 \%$, and $14.9 \%$, respectively (Figure 1 ).

The rates of patients treated with PIM of $0,1,2,3,4$, and $\geq 5$ before CGA were $20.8 \%(n=329), 42.0 \%(n=664)$, $13.9 \%(n=220), 9.3 \%(n=149), 6.5 \%(n=104)$, and $7.3 \%$ $(\mathrm{n}=116)$, respectively. The rates of patients with PPO of $0,1,2,3,4$, and $\geq 5$ after CGA were $25.4 \%(\mathrm{n}=402), 18.4 \%$ $(n=291), 28.0 \%(n=442), 16.78 \%(n=265), 7.6 \%(n=120)$, and $3.7 \%(n=59)$, respectively (Figure 2).
Table I Patients' characteristics

\begin{tabular}{|c|c|c|c|}
\hline & $\begin{array}{l}\text { Non-polypharmacy } \\
\text { (706) }\end{array}$ & $\begin{array}{l}\text { Polypharmacy } \\
(873)\end{array}$ & $p$-value \\
\hline Age (years) & $74.07 \pm 9.11$ & $77.28 \pm 8.45$ & 0.000 \\
\hline Female, n (\%) & $458(64.9 \%)$ & $566(63.6 \%)$ & 0.593 \\
\hline Education (years) & $7.12 \pm 4.67$ & $6.53 \pm 4.52$ & 0.025 \\
\hline \multicolumn{4}{|l|}{ Comorbidities (\%) } \\
\hline Depression & 28.1 & 34.1 & 0.013 \\
\hline $\begin{array}{l}\text { Cerebrovascular } \\
\text { disease }\end{array}$ & 5.2 & 12.0 & 0.000 \\
\hline Diabetes mellitus & 12.6 & 39.9 & 0.000 \\
\hline Hyperlipidemia & 13.7 & 22.5 & 0.000 \\
\hline Hypertension & 54.1 & 79.3 & 0.000 \\
\hline $\begin{array}{l}\text { Ischemic heart } \\
\text { disease }\end{array}$ & 9.2 & 29.8 & 0.000 \\
\hline $\begin{array}{l}\text { Congestive heart } \\
\text { failure }\end{array}$ & 2.8 & 11.3 & 0.000 \\
\hline $\begin{array}{l}\text { Peripheral artery } \\
\text { disease }\end{array}$ & 3.0 & 3.4 & 0.667 \\
\hline COPD & 3.4 & 13.3 & 0.000 \\
\hline Thyroid disease & 12.9 & 40.4 & 0.148 \\
\hline Osteoporosis & 19.3 & 22.1 & 0.196 \\
\hline Dementia & 12.2 & 20.9 & 0.000 \\
\hline Falls (\%) & 23.6 & 38.3 & 0.000 \\
\hline Charlson & $0.57 \pm 0.83$ & $1.35 \pm 1.08$ & 0.000 \\
\hline \multicolumn{4}{|l|}{ Comorbidity Index } \\
\hline \multicolumn{4}{|c|}{ Comprehensive geriatric assessment } \\
\hline MMSE & $24.75 \pm 5.90$ & $23.98 \pm 5.70$ & 0.070 \\
\hline COST & $23.36 \pm 5.48$ & $22.26 \pm 6.20$ & 0.062 \\
\hline MoCA & $24.12 \pm 4.12$ & $21.78 \pm 5.35$ & 0.000 \\
\hline YGDS & $3.14 \pm 3.44$ & $3.72 \pm 3.59$ & 0.008 \\
\hline Basic ADL & $90.87 \pm 16.88$ & $84.63 \pm 21.11$ & 0.000 \\
\hline Instrumental ADL & $12.13 \pm 5.13$ & $10.06 \pm 6.06$ & 0.000 \\
\hline MNA & $11.56 \pm 2.37$ & $10.87 \pm 2.52$ & 0.000 \\
\hline POMA-balance & $15.10 \pm 1.53$ & $13.69 \pm 2.48$ & 0.01 \\
\hline POMA-gait & $11.26 \pm 1.18$ & $10.58 \pm 1.72$ & 0.026 \\
\hline POMA-total & $26.36 \pm 2.55$ & $24.27 \pm 3.88$ & 0.002 \\
\hline Up\&Go (sec) & $10.54 \pm 3.44$ & $14.12 \pm 7.18$ & 0.002 \\
\hline \multicolumn{4}{|l|}{ Laboratory tests } \\
\hline Creatinine (mg/dL) & $0.84 \pm 0.28$ & $1.01 \pm 0.53$ & 0.000 \\
\hline $\begin{array}{l}\text { Thyroid stimulating } \\
\text { hormone (ulU/mL) }\end{array}$ & $2.22 \pm 6.31$ & $5.53 \pm 80.63$ & 0.332 \\
\hline Albumin & $4.04 \pm 0.48$ & $3.95 \pm 0.47$ & 0.002 \\
\hline 25(OH)D (ng/mL) & $18.50 \pm 14.52$ & $17.66 \pm 13.46$ & 0.289 \\
\hline Vitamin BI2 (pmol/L) & $426.63 \pm 333.209$ & $544.02 \pm 4|5.9|$ & 0.000 \\
\hline Folic acid (ng/mL) & $8.80 \pm 4.72$ & $8.77 \pm 5.39$ & 0.904 \\
\hline
\end{tabular}

Notes: COST: Cognitive State Test (0 [the worst]-30 [the best]); MMSE: MiniMental State Examination (0 [the worst]-30 [the best]); MNA: Mini-Nutritional Assessment (0 [the worst]-14 [the best]); MoCA: Montreal Cognitive Assessment (0 [the worst]-30 [the best]); Basic ADLs: Basic Activities of Daily Living (0 [the worst]- 100 [the best]); IADL: Instrumental Activities of Daily Living (0 [the worst]17 [the best]); POMA: Performance Oriented Mobility Assessment (0 [the worst]28 [the best]); YGDS: Yesavage Geriatric Depression Scale (I5 [the worst]-0 [the best]).

The drug group responsible for the highest increase in PPO was vitamin B12 and vitamin D. While 16.3\% $(n=246)$ of the patients received a single vitamin supplement (vitamin D or vitamin B12), 35.8\% ( $\mathrm{n}=538)$ of the patients received both vitamin $\mathrm{B}$ and vitamin $\mathrm{D}$ supplements. 


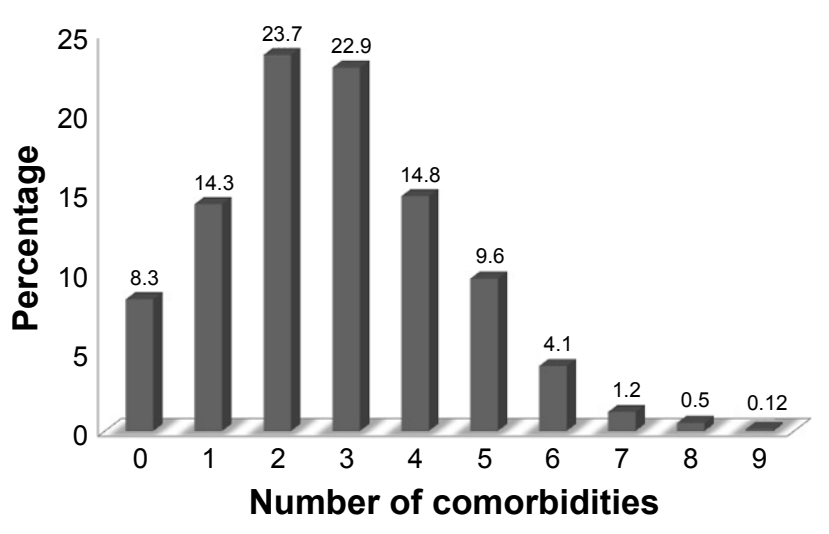

Figure I Number of comorbidities of the patients.

The drug groups which decreased, increased or had no change after CGA are shown in Tables 2 and 3. Accordingly, the drug groups with the highest decrease in use were in turn proton pump inhibitors (PPIs) by $6.9 \%(\mathrm{n}=109)$, dementia drugs by $6.9 \%(n=109)$, and antipsychotic drugs by $5.4 \%(\mathrm{n}=85)$. On the other hand, the drug groups with the highest increase in use were primarily vitamin supplements with vitamin $\mathrm{D}$ by $44.1 \%(\mathrm{n}=697)$ and vitamin $\mathrm{B}$ by $33.5 \%$ $(\mathrm{n}=530)$, secondly, anti-depressants by $14.1 \%(\mathrm{n}=223)$, and thirdly trazodone, which was used for the treatment of sleep disorders, by $12.8 \%(\mathrm{n}=202)$.

After CGA, monthly saved total per capita cost of PIMs was $\$ 12.8$ and monthly increased total per capita cost of PPOs was $\$ 5.6$. Among the PIMs, according to the total monthly cost, anti-dementia drugs were in the lead, accounting for $28.1 \%$ ( $\$ 5,697.9)$, followed by antipsychotics accounting for $12.5 \%(\$ 2,546.8)$, gastrointestinal system drugs accounting for $5.1 \%(\$ 1,038.2)$, and nonsteroidal anti-inflammatory drugs accounting for $4.5 \%(\$ 917.2)$.

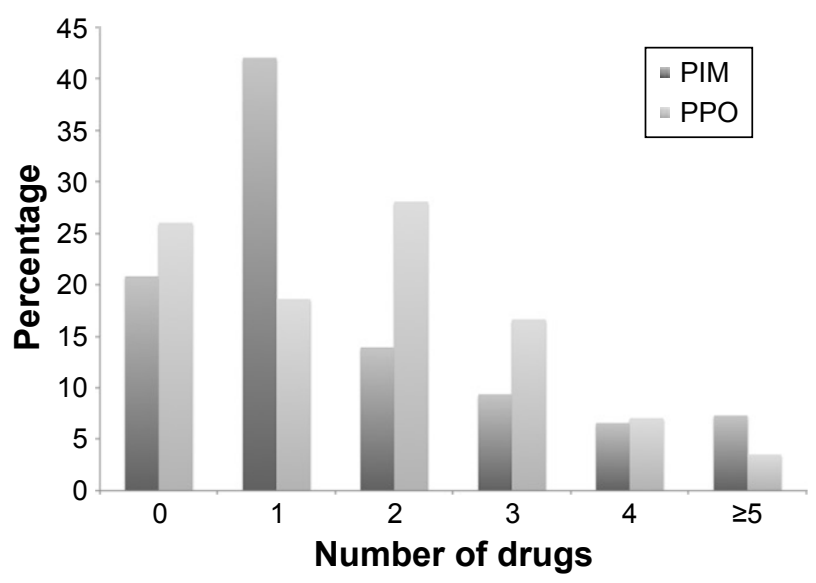

Figure 2 The rates of patients with PIMs and PPOs before CGA.

Abbreviations: CGA, comprehensive geriatric assessment; PIMs, potentially inappropriate medications; PPOs, potential prescribing omissions.
Table 2 The drug groups which decreased after CGA

\begin{tabular}{lll}
\hline & Before CGA & After CGA \\
\hline Alpha blockers & $6.9 \%(109)$ & $5.3 \%(84)$ \\
Angina pectoris medications & $11.5 \%(183)$ & $7.4 \%(I 18)$ \\
Anti-dementia drugs & $20.2 \%(320)$ & $13.7 \%(211)$ \\
Anti-epileptics & $2.7 \%(43)$ & $2.6 \%(42)$ \\
Antihypertensives & $56.1 \%(888)$ & $54.2 \%(859)$ \\
Antipsychotics & $9.4 \%(149)$ & $4.0 \%(64)$ \\
Benign prostatic hypertrophy & $3.2 \%(51)$ & $2.9 \%(46)$ \\
Beta blockers & $23.6 \%(374)$ & $20.2 \%(321)$ \\
Benzodiazepines & $2.28 \%(36)$ & $1.9 \%(30)$ \\
Chronic kidney disease & $1.20 \%(19)$ & $1.13 \%(18)$ \\
management drugs & & \\
Digoxin & $0.8 \%(14)$ & $0.4 \%(7)$ \\
Emesis treatment medications & $0.6 \%(10)$ & $0.1 \%(3)$ \\
Gingko biloba & $4.4 \%(71)$ & $0.5 \%(8)$ \\
HI receptor antagonists & $3.6 \%(58)$ & $1.6 \%(26)$ \\
Mixed insulins & $0.8 \%(13)$ & $0.75 \%(I 2)$ \\
Muscle relaxants & $0.6 \%(I 1)$ & $0.1 \%(3)$ \\
Neuropathy treatment drugs & $4.5 \%(72)$ & $3.4 \%(55)$ \\
Nonsteroidal anti- & $5.4 \%(86)$ & $1.5 \%(24)$ \\
inflammatory drugs & & \\
Oral antidiabetic agents & $18.3 \%(290)$ & $14.3 \%(277)$ \\
Osteoporosis medications & $6.9 \%(118)$ & $4.1 \%(66)$ \\
Proton pump inhibitors & $17.5 \%(277)$ & $10.6 \%(168)$ \\
Premeal insulins & $3.7 \%(60)$ & $2.4 \%(39)$ \\
Prokinetic agents & $4.74 \%(75)$ & $3.8 \%(61)$ \\
Urinary antiseptic drugs & $3.7 \%(59)$ & $2.4 \%(38)$ \\
Vertigo medications & $4.8 \%(76)$ & $2 \%(32)$ \\
Other drugs & $11.6 \%(I 85)$ & $4.6 \%(73)$ \\
\hline Ab &
\end{tabular}

Abbreviation: CGA, comprehensive geriatric assessment.

\section{Discussion}

The present study determined that polypharmacy was a common condition among older adults and that it might be associated with falls, depression, cognitive deficiency,

Table 3 The drug groups which increased after CGA

\begin{tabular}{lll}
\hline & Before CGA & After CGA \\
\hline Acetazolamide & $0.2 \%(4)$ & $0.4 \%(7)$ \\
Allopurinol & $1.01 \%(16)$ & $1.45 \%(23)$ \\
Analgesic medications & $8 \%(I 28)$ & $10.1 \%(160)$ \\
Anticoagulant medications & $5.2 \%(83)$ & $5.5 \%(88)$ \\
Antidepressant medications & $27.8 \%(440)$ & $40.2 \%(663)$ \\
Antithrombotic medications & $33.7 \%(534)$ & $36.4 \%(576)$ \\
Asthma treatment medications & $7.09 \%(I I 2)$ & $7.28 \%(I 15)$ \\
Dipeptidyl peptidase-4 inhibitors & $2.4 \%(38)$ & $10.1 \%(161)$ \\
Folic acid & $1.13 \%(30)$ & $5.19 \%(82)$ \\
Anti-hyperlipidemic & $16.5 \%(262)$ & $17.4 \%(276)$ \\
Parkinson's disease medications & $4.3 \%(69)$ & $5 \%(80)$ \\
Thyroid disease medications & $12.5 \%(198)$ & $13.4 \%(213)$ \\
Trazodone & $3.4 \%(54)$ & $16.1 \%(256)$ \\
Vitamin BI2 & $12.66 \%(200)$ & $46.23 \%(730)$ \\
Vitamin D & $5.50 \%(87)$ & $49.65 \%(784)$ \\
\hline Abbrevation
\end{tabular}

Abbreviation: CGA, comprehensive geriatric assessment. 
and decrease in functionality. In addition, it was also demonstrated that polypharmacy, number of the drugs used, and treatment cost could be reduced by the CGA including STOPP/START criteria.

The frequency of polypharmacy in older adults in Turkey was reported as $52.5 \% .^{8}$ In addition, considering the studies worldwide, the prevalence of polypharmacy was reported to be $76 \%$ in Australia, whereas it was reported as $53.6 \%$ with over-the-counter (OTC) medications and $26.7 \%$ without OTC medications in Germany. ${ }^{13,14}$ Consistent with the literature, the present study determined the rate of polypharmacy to be $55.3 \%$, which could be decreased to $34.4 \%$ after the CGA. It is obvious that prevention of polypharmacy in approximately $20 \%$ of the patients indicates how important CGA is. ${ }^{1,2,15-17}$ Furthermore, in geriatric patients with high health care expenditure, the impact of so-called prevention of polypharmacy on economic parameters cannot be ignored. In the present study, monthly per capita saving was approximately $\$ 12.8$ and annual per capita saving was approximately $\$ 153.46$ by exclusion of the PIMs after CGA. On the other hand, the monthly per capita saving was $\$ 5.68$ and annual per capita saving was $\$ 68.16$ after identification of PPOs by CGA. Thus, significant saving in health care expenditure is possible even with the PPOs after CGA. In addition, decreased treatment cost demonstrated herein is just the tip of the iceberg when the costs required for hospital stay, treatment, and drug-related morbidity and mortality are taken into account.

In the present study, the most frequent PIMs were PPIs, anti-dementia drugs, and antipsychotics. Inappropriate use of PPIs for gastric protection is quite common, and it is known that long-term PPI use may result in serious systemic side effects. These side effects include Clostridium difficile-associated colitis, community-acquired pneumonia, acute interstitial nephritis, vitamin B12 deficiency, increased risk of hip fractures, and increased incidence of fundic gland polyposis. ${ }^{17}$ In the present study, the second most frequently excluded PIMs were the anti-dementia drugs. The primary reason for this might be the fact that $\sim 5 \%-20 \%$ of the patients presenting to the geriatric clinics for memory impairment had curable causes. ${ }^{18}$ Among these causes, particularly hypothyroidism, vitamin B12 deficiency, depression, normal-pressure hydrocephalus, folic acid deficiency, and medications were reported most frequently. ${ }^{18}$ In addition, this outcome obtained from our reference center for dementia was highly important, as it revealed that many geriatric patients with misdiagnosed dementia had been administered anti-dementia medications by the clinics they had previously visited for subjective memory complaints, and that they might have been exposed to the side effects of those medications. ${ }^{19-22}$ The third most common PIMs were antipsychotics. This was attributed to the off-label use of antipsychotics, particularly in the treatment of behavioral and psychological symptoms of dementia as well as treatment of sleep disorders in geriatric patients. ${ }^{23}$ On the other hand, long-term use of high-dose antipsychotics may cause side effects including cardiovascular, metabolic, cognitive, and extrapyramidal. ${ }^{24}$

For this reason, antipsychotics carry a black box warning for increased risk of death in dementia patients (1.6-1.7 times higher than that of placebo), and they have been included in the American Geriatrics Society 2015 Updated Beers Criteria for Potentially Inappropriate Medication Use in Older Adults. ${ }^{24}$ Nevertheless, the recent guidelines have recommended low-dose antipsychotic use for less than 16 weeks in geriatric patients with behavioral symptoms of dementia when non-pharmacological approach remains inadequate. ${ }^{24}$ Considering potential complications of all of these three drug classes, it can be said that much higher cost saving than calculated, would be achieved.

On the other hand, vitamin D, vitamin B12, and folic acid preparations are the leading drugs among PPOs commenced after the CGA. Deficiency of micronutrients such as vitamin D, vitamin B12, and folic acid is prevalent in older adults and each may result in frailty, falls, depression, orthostatic hypotension, cognitive impairment, and increased frequency of depression and mortality. ${ }^{25-27}$ Therefore, these parameters are routinely evaluated within the context of CGA and appropriate micronutrient supplementation in patients with deficiency is of critical importance.

Other drugs with increased use after the CGA included anti-depressants and trazadone used for insomnia. This outcome indicated that depression and depression-associated insomnia might be overlooked and not be appropriately treated in older patients with pain, fragility, cognitive deficiency, numerous comorbidities, and polypharmacy. In fact, the frequency of geriatric depression in Turkey is $45.8 \%$ with the majority of patients remaining untreated. ${ }^{28}$ Appropriate treatment of geriatric depression, which may have devastating consequences such as falls, frailty, sleep disorders, cognitive deficiency, malnutrition, self-negligence, and increased risk of morbidity and mortality, may prevent polypharmacy by reducing somatic complaints, which health care professionals try to treat, but which are actually related to depression. ${ }^{29}$ 
The strengths of the present study include large sample size; evaluating laboratory findings, sociodemographic characteristics, comorbidities, and all subgroups of drugs separately; and performing the CGA complemented with STOPP/START criteria in each patient. However, the retrospective design of the study, not evaluating long-term effects of the new treatment planned after the CGA on the CGA parameters including falls, depression, cognitive and functional capacity, and comprising only ambulatory patients may be among the limitations.

Polypharmacy is a common condition among geriatric patients and is associated with falls, depression, cognitive deficiency, and decreased functionality. In the presence of numerous comorbidities and geriatric syndromes, changes in pharmacokinetics and pharmacodynamics and in tolerability of potential drug side effects due to physiological changes with aging lead to difficulties in planning treatments of such patients appropriately. For this reason, performing a CGA including STOPP/START criteria is quite important. Thus, long-term complications would be prevented by avoiding PIMs and polypharmacy, and allowing the use of correct drugs identified by PPOs that may be balanced with the potential cost of disease burden avoided. As a result, decreased drug-related costs in older adults clearly demonstrate both medical and economic benefits of CGA.

\section{Disclosure}

The authors report no conflicts of interest in this work.

\section{References}

1. World Health Organization. World Health Statistics, 2013. Geneva: World Health Organization. Available from: http://www.who.int/gho/ publications/world_health_statistics/2013/en/.

2. Wilson M, Mair A, Dreischulte T, Witham MD; NHS Scotland Model of Care Polypharmacy Working Group. Prescribing to fit the needs of older people the - NHS Scotland Polypharmacy Guidance, 2nd edition. $J$ R Coll Physicians Edinb. 2015;45(2):108-113.

3. Onder G, Landi F, Fusco D, et al. Recommendations to prescribe in complex older adults: results of the CRIteria to assess appropriate Medication use among Elderly complex patients (CRIME) project. Drugs Aging. 2014;31(1):33-45.

4. Johnson JA, Bootman JL. Drug-related morbidity and mortality. A costof-illness model. Arch Intern Med. 1995;155(18):1949-1956.

5. Ernst FR, Grizzle AJ. Drug-related morbidity and mortality: updating the cost-of-illness model. J Am Pharm Assoc (Wash). 2001;41(2):192-199.

6. Smith DL. The effect of patient non-compliance on health care costs. Med Interface. 1993;6(4):74-76.

7. Sullivan SD, Kreglig DH, Hazleet TK. Noncompliance with medication regimens and subsequent hospitalization: a literature analysis and cost of hospitalization estimate. J Res Pharm Econ. 1990;2(2): 19-33.

8. Bahat G, Bay I, Tufan A, et al. Prevalence of potentially inappropriate prescribing among older adults: a comparison of the Beers 2012 and Screening Tool of Older Person's Prescriptions criteria version 2. Geriatr Gerontol Int. 2017;17(9):1245-1251.
9. O’Mahony D, O'Sullivan D, Byrne S, et al. STOPP/START criteria for potentially inappropriate prescribing in older people: version 2 . Age Ageing. 2015;44(2):213-218.

10. Babacan-Yildiz G, Isik AT, Ur E, et al. COST: Cognitive State Test, a brief screening battery for Alzheimer disease in illiterate and literate patients. Int Psychogeriatr. 2013;25(3):403-412.

11. Soysal P, Isik AT, Buyukaydin B, Kazancioglu R. A comparison of end-stage renal disease and Alzheimer's disease in the elderly through a comprehensive geriatric assessment. Int Urol Nephrol. 2014;46(8): $1627-1632$.

12. Jyrkka J, Enlund H, Korhonen MJ, Sulkava R, Hartikainen S. Polypharmacy status as an indicator of mortality in an elderly population. Drugs Aging. 2009;26(12):1039-1048.

13. Hubbard RE, Peel NM, Scott IA, et al. Polypharmacy among inpatients aged 70 years or older in Australia. Med J Aust. 2015;202(7): 373-377.

14. Junius-Walker U, Theile G, Hummers-Pradier E. Prevalence and predictors of polypharmacy among older primary care patients in Germany. Fam Pract. 2007;24(1):14-19.

15. Moorhouse P, Mallery LH. Palliative and therapeutic harmonization: a model for appropriate decision-making in frail older adults. $J \mathrm{Am}$ Geriatr Soc. 2012;60(12):2326-2332.

16. Beers MH, Ouslander JG, Rollingher I, et al. Explicit criteria for determining inappropriate medication use in nursing home residents. UCLA Division of Geriatric Medicine. Arch Intern Med. 1991;151(9): $1825-1832$.

17. Masclee GM, Sturkenboom MC, Kuipers EJ. A benefit-risk assessment of the use of proton pump inhibitors in the elderly. Drugs Aging. 2014;31(4):263-282.

18. Muangpaisan W, Petcharat C, Srinonprasert V. Prevalence of potentially reversible conditionsin dementia and mild cognitive impairment in a geriatric clinic. Geriatr Gerontol Int. 2012;12(1):59-64.

19. Soysal P, Isik AT. Effects of acetylcholinesterase inhibitors on nutritional status in elderly patients with dementia: a 6-month follow-up study. J Nutr Health Aging. 2016;20(4):398-403.

20. Soysal P, Isik AT, Stubbs B. Acetylcholinesterase inhibitors are associated with weight loss in older people with dementia: a systematic review and meta-analysis. J Neurol Neurosurg Psychiatry. 2016;87(12): $1368-1374$.

21. Isik AT, Soysal P, Usarel C. Effects of acetylcholinesterase inhibitors on balance and gait functions and orthostatic hypotension in elderly patients with Alzheimer disease. Am J Alzheimers Dis Other Demen. 2016;31(7):580-584.

22. Soysal P, Isik AT. Asymptomatic bradycardia due to rivastigmine in an elderly adult with Lewy body dementia. J Am Geriatr Soc. 2015; 63(12):2648-2650.

23. Schroeck JL, Ford J, Conway EL, et al. Review of safety and efficacy of sleep medicines in older adults. Clin Ther. 2016;38(11): 2340-2372.

24. American Geriatrics Society 2012 Beers Criteria Update Expert Panel. American Geriatrics Society updated Beers Criteria for potentially inappropriate medication use in older adults. J Am Geriatr Soc. 2012; 60(4):616-631.

25. Soysal P, Yay A, Isik AT. Does vitamin D deficiency increase orthostatic hypotension risk in the elderly patients? Arch Gerontol Geriatr. 2014; 59(1):74-77.

26. Bulut EA, Soysal P, Aydin AE, et al. Vitamin B12 deficiency might be related to sarcopenia in older adults. Exp Gerontol. 2017;95: 136-140.

27. Ometto F, Stubbs B, Annweiler C, et al. Hypovitaminosis D and orthostatic hypotension: a systematic review and meta-analysis. J Hypertens. 2016;34(6):1036-1043.

28. Arslantas D, Ünsal A, Ozbabalık D. Prevalence of depression and associated risk factors among the elderly in Middle Anatolia, Turkey. Geriatr Gerontol Int. 2014;14(1):100-108.

29. Soysal P, Veronese N, Thompson T, et al. Relationship between depression and frailty in older adults: a systematic review and meta-analysis. Ageing Res Rev. 2017;36:78-87. 


\section{Publish your work in this journal}

Clinical Interventions in Aging is an international, peer-reviewed journal focusing on evidence-based reports on the value or lack thereof of treatments intended to prevent or delay the onset of maladaptive correlates of aging in human beings. This journal is indexed on PubMed Central, MedLine,
Dovepress

CAS, Scopus and the Elsevier Bibliographic databases. The manuscript management system is completely online and includes a very quick and fair peer-review system, which is all easy to use. Visit http://www.dovepress. $\mathrm{com} /$ testimonials.php to read real quotes from published authors. 